\title{
Molecular characterization of H3N2 influenza A viruses isolated from Ontario swine in 2011 and 2012
}

Helena Grgic ${ }^{1,5^{*}}$, Marcio Costa ${ }^{2,5}$, Robert M Friendship ${ }^{1,5}$, Susy Carman ${ }^{3}$, Éva Nagy ${ }^{2,5}$, Greg Wideman ${ }^{4}$, Scott Weese $\mathrm{C}^{2,5}$ and Zvonimir Poljak ${ }^{1,5}$

\begin{abstract}
Background: Data about molecular diversity of commonly circulating type A influenza viruses in Ontario swine are scarce. Yet, this information is essential for surveillance of animal and public health, vaccine updates, and for understanding virus evolution and its large-scale spread.

Methods: The study population consisted of 21 swine herds with clinical problems due to respiratory disease. Nasal swabs from individual pigs were collected and tested by virus isolation in MDCK cells and by rtRT-PCR. All eight segments of $10 \mathrm{H} 3 \mathrm{~N} 2$ viruses were sequenced using high-throughput sequencing and molecularly characterized.

Results: Within-herd prevalence ranged between 2 and 100\%. Structurally, Ontario H3N2 viruses could be classified into three different groups. Group 1 was the most similar to the original trH3N2 virus from 2005. Group 2 was the most similar to the Ontario turkey H3N2 isolates with PB1 and NS genes originating from trH3N2 virus and M, PB2, $\mathrm{PA}$ and NP genes originating from the $\mathrm{A}(\mathrm{H} 1 \mathrm{~N} 1) \mathrm{pdm} 09$ virus. All Group 3 internal genes were genetically related to $\mathrm{A}(\mathrm{H} 1 \mathrm{N1}$ )pdm09. Analysis of antigenic sites of HA1 showed that Group 1 had 8 aa changes within 4 antigenic sites, $A(1), B(3), C(2)$ and $E(2)$. The Group 2 viruses had 8 aa changes within 3 antigenic sites $A(3), B(3)$ and $C(2)$, while Group 3 viruses had 4 aa changes within 3 antigenic sites, $B(1), D(1)$ and $E(2)$, when compared to the cluster IV H3N2 virus [A/swine/Ontario/33853/2005/(H3N2)].
\end{abstract}

Conclusions: The characterization of the Ontario H3N2 viruses clearly indicates reassortment of gene segments between the North American swine trH3N2 from cluster IV and the A(H1N1)pdm09 virus.

Keywords: Influenza A virus, Triple-reassortant H3N2, Swine, 454 sequencing, Phylogenetic analysis

\section{Background}

Influenza viruses, belonging to the family Orthomyxoviridae, are enveloped viruses with segmented negative-sense RNA genome [1]. Influenza A viruses evolve rapidly, creating new variants which could be the result of either point mutations or reassortment. Eighteen hemagglutinin (HA) and 11 neuraminidase (NA) types have been reported to date, classifying viruses into subtypes $\mathrm{H} 1$ to $\mathrm{H} 18$ and $\mathrm{N} 1$ to N11 [2].

\footnotetext{
* Correspondence: hgrgic@uoguelph.ca

'Department of Population Medicine, Ontario Veterinary College, University

of Guelph, Guelph, Ontario N1G 2 W1, Canada

${ }^{5}$ Centre for Public Health and Zoonoses, University of Guelph, Guelph,

Canada

Full list of author information is available at the end of the article
}

In 2005, the triple-reassortant H3N2 (trH3N2) virus was reported in Canada and spread widely, affecting swine industries in all provinces [3]. After initial detection of the trH3N2 virus in 2005, there were no further scientific publications about molecular diversity of influenza viruses circulating in Canadian swine until 2009 [4]. This information has been particularly limited in Ontario. According to a recent statistic from 2012 and 2013, Ontario is the province with the second largest number of pigs on farms in Canada, and with the largest number of farms with pigs [5]. This information is important for surveillance of influenza viruses and informing animal and public health decisions, vaccine updates, and for understanding virus evolution and its large-scale spread. Therefore, the objective of this study

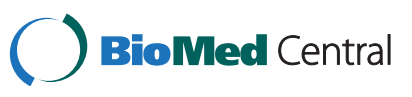

(c) 2014 Grgić et al.; licensee BioMed Central Ltd. This is an Open Access article distributed under the terms of the Creative Commons Attribution License (http://creativecommons.org/licenses/by/4.0), which permits unrestricted use, distribution, and reproduction in any medium, provided the original work is properly credited. The Creative Commons Public Domain Dedication waiver (http://creativecommons.org/publicdomain/zero/1.0/) applies to the data made available in this article, unless otherwise stated. 
was to determine which H3N2 influenza A viruses circulated in Ontario swine in 2011 and 2012.

\section{Results}

\section{Descriptive analysis}

Most of the 21 herds included in this study were finisher sites only $(n=9)$, followed by nursery $(n=6)$, wean-tofinish $(n=3)$, farrow-to-finish $(n=1)$, farrow-to-grow $(n=1)$, and farrow-to-wean $(\mathrm{n}=1)$ sites. Sow capacity ranged between 600 and 650 sows. Nursery inventory at the date of sampling ranged between 2000 and 2500 animals, and finisher pig inventory ranged between 950 and 5000 animals. The average number of samples tested per herd was 56 (7-100), and the average number of pooled real-time reverse transcription PCR (rtRT-PCR) tests was 10 (5-20) per herd. The mean number of sampled animals was 53 and 57.3 animals in influenza-virus-negative and influenzavirus-positive herds, respectively $(\mathrm{p}=0.7)$.

In this study, 13 of 21 herds (61.9\%) tested positive for influenza virus using virus isolation in Madin-Darby canine kidney (MDCK) cells, while 8 of 12 herds (66.7\%) tested rtRT-PCR-positive on pooled samples. In total, 16 herds $(76.2 \%)$ tested positive by either virus isolation or rtRT-PCR. Viruses from eleven out of 16 positive herds were typed as H3N2, from 3 herds were typed as H1N1, and in 2 herds, subtyping was not successful. In total 11 H3N2 isolates from 10 different herds were included for full genome sequencing in 2 separate runs. However, sequencing results for 10 isolates from 9 herds were obtained. Full genome sequencing results for one virus could not be obtained despite repeated inclusion of this virus in two separate runs.
Figure 1 depicts the within-herd prevalence of influenza virus shedding in 21 Ontario swine herds tested by MDCK and rtRT-PCR. The within-herd prevalence ranged between $2 \%$ and $100 \%$. In herds that had at least one virus positive isolation, the mean within-herd prevalence was $84 \%$ in sow herds $(n=2), 46 \%$ in nursery herds $(n=6)$, $30 \%$ in finisher herds $(n=4)$, and $57 \%$ in wean-to-finish herds $(n=1)$.

\section{Genetic characterization of 10 Ontario H3N2 influenza viruses}

Structurally, Ontario H3N2 viruses detected in this study could be classified into three groups. The first group consisted of 4 viruses in which all segments were similar to those of the trH3N2 virus that emerged in Ontario in 2005 [3]. The second group consisted of 4 viruses with HA, NA, PB1 and NS genes originating from the trH3N2 virus, and $\mathrm{M}, \mathrm{PB} 2, \mathrm{PA}$ and $\mathrm{NP}$ genes originating from the pandemic $\mathrm{A}(\mathrm{H} 1 \mathrm{~N} 1) \mathrm{pdm} 09$ virus. The third group consisted of 2 viruses with HA and NA genes originating from the trH3N2 virus, and with all internal genes originating from the $\mathrm{A}(\mathrm{H} 1 \mathrm{~N} 1) \mathrm{pdm} 09$ virus. The phylogenetic relationships of the HA gene of these Ontario H3N2 viruses are presented, in association with other reference viruses in Figure 2. Genome constellations identified in Ontario H3N2 viruses isolated from swine in 2011 and 2012 are presented in Additional file 1. The GenBank accession numbers of the sequences reported in this paper are: [KF840476-KF840478, KJ413857-KJ413933].

More detailed analysis of HA and NA genes revealed that Group 1 indeed was the most similar to the original Cluster IV trH3N2 [A/swine/ON/33853/2005 (H3N2] virus from

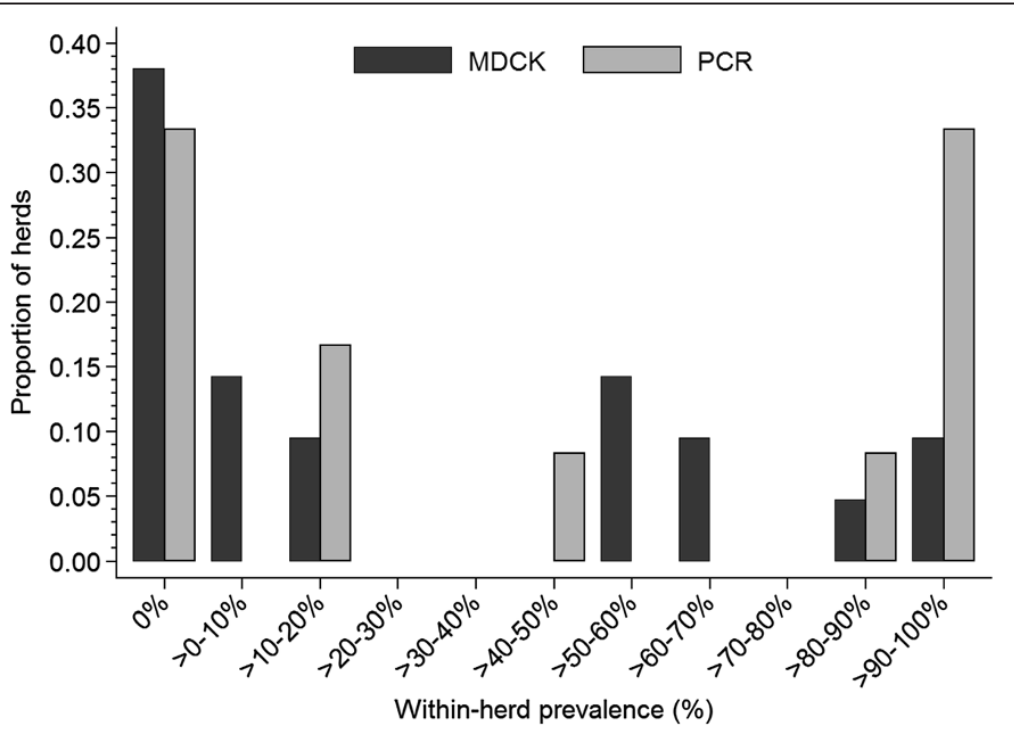

Figure 1 Within-herd prevalence of positive samples for influenza virus during testing of individual samples by virus isolation in Madin-Darby canine kidney (MDCK) cells, and during testing of 3:1 pools of nasal swabs on real-time reverse transcription (rtRT)-PCR in Ontario swine herds during 2011-2012. 


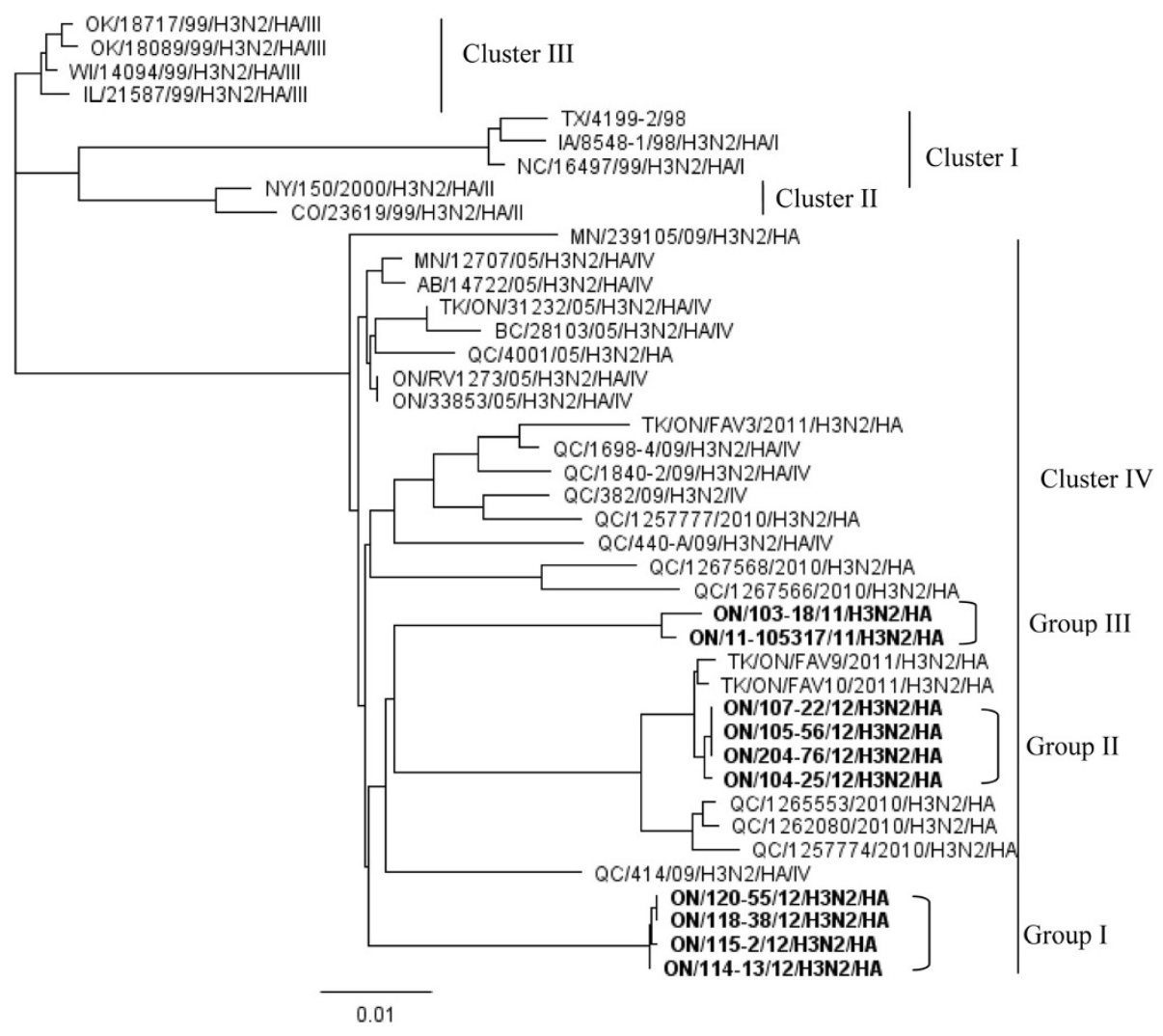

Figure 2 Phylogenetic tree of the HA gene nucleotide sequences of 10 Ontario H3N2 viruses.

2005, with $97.2 \%$ to $97.3 \%$ and $97.7 \%$ to $97.8 \%$ identity of $\mathrm{HA}$ and NA genes, respectively (Tables 1 and 2). Interestingly, when compared to published standards, the NA gene of these Group 1 viruses showed the highest identity (at 98.3\% to $98.7 \%$ ) (Table 2) with the two 2011 Ontario turkey isolates, while the HA gene of 4 Group 1 viruses showed much more modest identity with these 2 turkey viruses (94.6\% to $94.7 \%$ ) (Table 1 ). Group 1 was a homogenous group of 4 viruses, with $99.9 \%$ to $100 \%$ (HA) and $99.7 \%$ to $100 \%$ (NA) identity of the HA and NA genes, although these herds were not a part of the same production system.

More detailed analysis of HA and NA genes revealed that the 4 Group 2 viruses could also be grouped with Cluster IV viruses. Group 2 was most similar to the 2 Ontario turkey H3N2 variants detected in 2011 [6], with $99.6 \%$ to $99.7 \%$ and $99.4 \%$ to $99.6 \%$ identity on HA (Table 1) and NA (Table 2) genes, respectively. Interestingly, when compared to published standards, the HA gene of these 4 Group 2 viruses showed a high identity (at 98.6\% to 98.8\%) (Table 1) with the 2010 Quebec swine isolates. However, the NA gene of viruses showed an identity of only $95.8 \%$ to $96.0 \%$ with these Quebec swine viruses (Table 2). Group 2 was also a homogenous group of viruses with $99.9 \%$ to $100 \%$ and $99.9 \%$ to $100 \%$ identity of the HA and NA genes, respectively, although these herds were not a part of the same production system.

More detailed analysis of HA and NA genes revealed that the 2 Group 3 viruses could also be grouped with Cluster IV viruses. Group 3 viruses were most similar to the original trH3N2 [A/turkey/ON/31232/2005 and A/ swine/ON/33853/2005(H3N3)] viruses detected in 2005 [3], with $96.8 \%$ to $97.1 \%$ and $98 \%$ to $98.1 \%$ identity of HA and NA genes, respectively. Interestingly, when compared to published standards, the HA and NA genes of this group of viruses showed maximum identity of $95.9 \%$ and 97.4\% of the HA and NA gene, respectively, with any of the recent Canadian isolates available at the time of this study (Tables 1 and 2). This was also a homogenous group of viruses, with $99.5 \%$ and $99.9 \%$ identity of HA and NA genes, respectively, but both isolates were from a single herd.

The relatedness of each internal gene segment from each of the 10 Ontario H3N2 viruses was compared with published influenza A virus sequences. More detailed analysis of internal genes showed that the 4 Group 1 viruses indeed were the most similar to the original trH3N2 virus from 2005. The 4 Group 2 viruses were most similar to the Ontario turkey $\mathrm{H} 3 \mathrm{~N} 2$ variants with $\mathrm{PB} 1$ and NS genes originating from trH3N2 virus and $\mathrm{M}, \mathrm{PB} 2, \mathrm{PA}$, and $\mathrm{NP}$ 
Table 1 Identity table of the full length of the HA gene of 10 Ontario H3N2 viruses isolated from swine in 2011-2012 and selected references viruses based on nucleotide sequence

\begin{tabular}{|c|c|c|c|c|c|c|c|c|c|c|}
\hline & $\begin{array}{l}\text { G1-A/sw/ } \\
\text { ON/114- } \\
\text { 13/12/ } \\
\text { H3N2/HA }\end{array}$ & $\begin{array}{l}\text { G1-A/sw/ } \\
\text { ON/115-2/ } \\
12 / \mathrm{H} 3 \mathrm{~N} 2 / \\
\mathrm{HA}\end{array}$ & $\begin{array}{l}\text { G1-A/sw/ } \\
\text { ON/118- } \\
38 / 12 / \\
\text { H3N2/HA }\end{array}$ & $\begin{array}{l}\text { G1-A/sw/ } \\
\text { ON/120- } \\
55 / 12 / \\
\text { H3N2/HA }\end{array}$ & $\begin{array}{l}\text { G2-A/sw/ } \\
\text { ON/104- } \\
25 / 12 / \\
\text { H3N2/HA }\end{array}$ & $\begin{array}{l}\text { G2-A/sw/ } \\
\text { ON/204- } \\
76 / 12 / \\
\text { H3N2/HA }\end{array}$ & $\begin{array}{l}\text { G2-A/sw/ } \\
\text { ON/105- } \\
56 / 12 / \\
\text { H3N2/HA }\end{array}$ & $\begin{array}{l}\text { G2-A/sw/ } \\
\text { ON/107- } \\
22 / 12 / \\
\text { H3N2/HA }\end{array}$ & $\begin{array}{l}\text { G3-A/sw/ } \\
\text { ON/103- } \\
18 / 11 / \\
\text { H3N2/HA }\end{array}$ & $\begin{array}{l}\text { G3-A/sw/ } \\
\text { ON/11- } \\
105317 / \\
11 / \mathrm{H} 3 / \mathrm{HA}\end{array}$ \\
\hline $\begin{array}{l}\text { G1-A/sw/ } \\
\text { ON/114-13/ } \\
12 / H 3 N 2 / \\
\text { HA }\end{array}$ & 100 & 99.9 & 99.9 & 99.9 & 94.8 & 94.8 & 94.8 & 94.8 & 94.9 & 95.2 \\
\hline $\begin{array}{l}\text { G1-A/sw/ } \\
\text { ON/115-2/ } \\
12 / H 3 N 2 / \\
\text { HA }\end{array}$ & 99.9 & 100 & 99.9 & 99.9 & 94.7 & 94.7 & 94.7 & 94.7 & 94.9 & 95.1 \\
\hline $\begin{array}{l}\text { G1-A/sw/ } \\
\text { ON/118-38/ } \\
12 / H 3 N 2 / \\
\text { HA }\end{array}$ & 99.9 & 99.9 & 100 & 100 & 94.7 & 94.7 & 94.7 & 94.7 & 94.9 & 95.1 \\
\hline $\begin{array}{l}\text { G1-A/sw/ } \\
\text { ON/120-55/ } \\
12 / H 3 N 2 / \\
\text { HA }\end{array}$ & 99.9 & 99.9 & 100 & 100 & 94.7 & 94.7 & 94.7 & 94.7 & 94.9 & 95.1 \\
\hline $\begin{array}{l}\text { G2-A/sw/ } \\
\text { ON/104-25/ } \\
12 / H 3 N 2 / \\
\text { HA }\end{array}$ & 94.8 & 94.7 & 94.7 & 94.7 & 100 & 99.9 & 99.9 & 99.9 & 94.9 & 94.9 \\
\hline $\begin{array}{l}\text { G2-A/sw/ } \\
\text { ON/204-76/ } \\
12 / H 3 N 2 / \\
\text { HA }\end{array}$ & 94.8 & 94.7 & 94.7 & 94.7 & 99.9 & 100 & 100 & 100 & 94.8 & 94.9 \\
\hline $\begin{array}{l}\text { G2-A/sw/ } \\
\text { ON/105-56/ } \\
12 / H 3 N 2 / \\
\text { HA }\end{array}$ & 94.8 & 94.7 & 94.7 & 94.7 & 99.9 & 100 & 100 & 100 & 94.8 & 94.9 \\
\hline $\begin{array}{l}\text { G2-A/sw/ } \\
\text { ON/107-22/ } \\
12 / H 3 N 2 / \\
\text { HA }\end{array}$ & 94.8 & 94.7 & 94.7 & 94.7 & 99.9 & 100 & 100 & 100 & 94.8 & 94.9 \\
\hline $\begin{array}{l}\text { G3-A/sw/ } \\
\text { ON/103-18/ } \\
11 / H 3 N 2 / \\
\text { HA }\end{array}$ & 94.9 & 94.9 & 94.9 & 94.9 & 94.9 & 94.8 & 94.8 & 94.8 & 100 & 99.5 \\
\hline $\begin{array}{l}\text { G3-A/sw/ } \\
\text { ON/11- } \\
\text { 105317/11/ } \\
\text { H3N2/HA }\end{array}$ & 95.2 & 95.1 & 95.1 & 95.1 & 94.9 & 94.9 & 94.9 & 94.9 & 99.5 & 100 \\
\hline $\begin{array}{l}\text { MN/12707/ } \\
\text { 05/H3N2/ } \\
\text { HA/IV }\end{array}$ & 97 & 96.9 & 96.9 & 96.9 & 96.5 & 96.5 & 96.5 & 96.5 & 96.5 & 96.8 \\
\hline $\begin{array}{l}\text { AB/14722/ } \\
05 / \text { H3N2/ } \\
\text { HA/IV }\end{array}$ & 97 & 96.9 & 96.9 & 96.9 & 96.5 & 96.5 & 96.5 & 96.5 & 96.5 & 96.8 \\
\hline $\begin{array}{l}\text { TK/ON/ } \\
31232 / 05 / \\
\text { H3N2/HA/IV }\end{array}$ & 96.9 & 96.8 & 96.8 & 96.8 & 96.4 & 96.4 & 96.4 & 96.4 & 96.4 & 96.6 \\
\hline $\begin{array}{l}\text { BC/28103/ } \\
05 / H 3 N 2 / \\
\text { HA/IV }\end{array}$ & 96.5 & 96.4 & 96.4 & 96.4 & 96 & 96 & 96 & 96 & 95.9 & 96.2 \\
\hline $\begin{array}{l}\mathrm{QC} / 4001 / \\
05 / \mathrm{H} 3 \mathrm{~N} 2 / \\
\mathrm{HA}\end{array}$ & 96.6 & 96.5 & 96.5 & 96.5 & 96.1 & 96.1 & 96.1 & 96.1 & 96.1 & 96.4 \\
\hline
\end{tabular}


Table 1 Identity table of the full length of the HA gene of 10 Ontario H3N2 viruses isolated from swine in 2011-2012 and selected references viruses based on nucleotide sequence (Continued)

\begin{tabular}{|c|c|c|c|c|c|c|c|c|c|c|}
\hline $\begin{array}{l}\text { ON/ } \\
\text { RV1273/05/ } \\
\text { H3N2/HA/IV }\end{array}$ & 97.3 & 97.2 & 97.2 & 97.2 & 96.8 & 96.8 & 96.8 & 96.8 & 96.8 & 97.1 \\
\hline $\begin{array}{l}\text { ON/33853/ } \\
\text { 05/H3N2/ } \\
\text { HA/IV }\end{array}$ & 97.3 & 97.2 & 97.2 & 97.2 & 96.8 & 96.8 & 96.8 & 96.8 & 96.8 & 97.1 \\
\hline $\begin{array}{l}\text { QC/1698-4/ } \\
09 / H 3 N 2 / \\
\text { HA/IV }\end{array}$ & 96.1 & 96.1 & 96.1 & 96.1 & 95.5 & 95.5 & 95.5 & 95.5 & 95.6 & 95.9 \\
\hline $\begin{array}{l}\text { QC/1840-2/ } \\
09 / H 3 N 2 / \\
\text { HA/IV }\end{array}$ & 95.9 & 95.9 & 95.9 & 95.9 & 95.4 & 95.4 & 95.4 & 95.4 & 95.6 & 95.8 \\
\hline $\begin{array}{l}\text { QC/382/09/ } \\
\text { H3N2/IV }\end{array}$ & 96.1 & 96 & 96 & 96 & 95.5 & 95.5 & 95.5 & 95.5 & 95.5 & 95.7 \\
\hline $\begin{array}{l}\text { QC/ } \\
1257777 / \\
2010 / \mathrm{H} 3 \mathrm{~N} 2 / \\
\mathrm{HA}\end{array}$ & 95.8 & 95.8 & 95.8 & 95.8 & 95.2 & 95.2 & 95.2 & 95.2 & 95.2 & 95.4 \\
\hline $\begin{array}{l}\text { QC/440-A/ } \\
09 / H 3 N 2 / \\
\text { HA/IV }\end{array}$ & 95.6 & 95.6 & 95.6 & 95.6 & 95 & 95 & 95 & 95 & 95.2 & 95.4 \\
\hline $\begin{array}{l}\text { MN/ } \\
239105 / 09 / \\
\text { H3N2/HA }\end{array}$ & 95.8 & 95.8 & 95.8 & 95.8 & 95.3 & 95.3 & 95.3 & 95.3 & 95.5 & 95.8 \\
\hline $\begin{array}{l}\text { QC/414/09/ } \\
\text { H3N2/HA/IV }\end{array}$ & 95.7 & 95.6 & 95.6 & 95.6 & 95.4 & 95.4 & 95.4 & 95.4 & 95.7 & 95.9 \\
\hline $\begin{array}{l}\text { QC/ } \\
1267568 / \\
2010 / H 3 N 2 / \\
\text { HA }\end{array}$ & 95.2 & 95.1 & 95.1 & 95.1 & 94.6 & 94.6 & 94.6 & 94.6 & 94.8 & 95.1 \\
\hline $\begin{array}{l}\text { QC/ } \\
1267566 / \\
2010 / H 3 N 2 / \\
\text { HA }\end{array}$ & 95.1 & 95 & 95 & 95 & 94.3 & 94.3 & 94.3 & 94.3 & 94.3 & 94.5 \\
\hline $\begin{array}{l}\text { TK/ON/ } \\
\text { FAV9/2011/ } \\
\text { H3N2/HA }\end{array}$ & 94.7 & 94.7 & 94.7 & 94.7 & 99.7 & 99.7 & 99.7 & 99.7 & 94.8 & 94.9 \\
\hline $\begin{array}{l}\text { TK/ON/ } \\
\text { FAV10/ } \\
2011 / \mathrm{H} 3 \mathrm{~N} 2 / \\
\text { HA }\end{array}$ & 94.7 & 94.6 & 94.6 & 94.6 & 99.6 & 99.6 & 99.6 & 99.6 & 94.8 & 94.9 \\
\hline $\begin{array}{l}\text { QC/ } \\
1265553 / \\
2010 / H 3 N 2 / \\
\text { HA }\end{array}$ & 94.6 & 94.6 & 94.6 & 94.6 & 98.6 & 98.6 & 98.6 & 98.6 & 94.8 & 94.9 \\
\hline $\begin{array}{l}\mathrm{QC} / \\
1262080 / \\
2010 / \mathrm{H} 3 \mathrm{~N} 2 / \\
\mathrm{HA}\end{array}$ & 94.5 & 94.5 & 94.5 & 94.5 & 98.8 & 98.8 & 98.8 & 98.8 & 94.6 & 94.8 \\
\hline $\begin{array}{l}\text { QC/ } \\
1257774 / \\
2010 / \mathrm{H} 3 \mathrm{~N} 2 / \\
\mathrm{HA}\end{array}$ & 94.6 & 94.5 & 94.5 & 94.5 & 98.7 & 98.7 & 98.7 & 98.7 & 94.4 & 94.6 \\
\hline $\begin{array}{l}\text { OK/18717/ } \\
99 / \mathrm{H} 3 \mathrm{~N} 2 / \\
\text { HA/III }\end{array}$ & 94.2 & 94.2 & 94.1 & 94.1 & 93.7 & 93.8 & 93.8 & 93.8 & 93.7 & 93.9 \\
\hline $\begin{array}{l}\text { OK/18089/ } \\
99 / \mathrm{H} 32 / \\
\text { HA/III }\end{array}$ & 94.2 & 94.1 & 94.1 & 94.1 & 93.6 & 93.8 & 93.8 & 93.8 & 93.7 & 94 \\
\hline
\end{tabular}


Table 1 Identity table of the full length of the HA gene of 10 Ontario H3N2 viruses isolated from swine in 2011-2012 and selected references viruses based on nucleotide sequence (Continued)

\begin{tabular}{|c|c|c|c|c|c|c|c|c|c|c|}
\hline $\begin{array}{l}\text { WI/14094/ } \\
\text { 99/H3N2/ } \\
\text { HA/III }\end{array}$ & 94.2 & 94.2 & 94.2 & 94.2 & 93.9 & 94 & 94 & 94 & 93.7 & 94 \\
\hline $\begin{array}{l}\text { IL/21587/ } \\
99 / \mathrm{H} 3 \mathrm{~N} 2 / \\
\mathrm{HA} / \mathrm{III}\end{array}$ & 94.1 & 94.1 & 94.1 & 94.1 & 93.8 & 93.9 & 93.9 & 93.9 & 93.6 & 93.9 \\
\hline $\begin{array}{l}\text { TX/4199-2/ } \\
98\end{array}$ & 90.4 & 90.3 & 90.3 & 90.3 & 90.1 & 90.3 & 90.3 & 90.3 & 89.4 & 89.7 \\
\hline $\begin{array}{l}\text { IA/8548-1/ } \\
98 / \mathrm{H} 3 \mathrm{~N} 2 / \\
\mathrm{HA} / \mathrm{I}\end{array}$ & 90.5 & 90.4 & 90.4 & 90.4 & 90.2 & 90.4 & 90.4 & 90.4 & 89.4 & 89.7 \\
\hline $\begin{array}{l}\text { NC/16497/ } \\
\text { 99/H3N2/ } \\
\text { HA/I }\end{array}$ & 90.7 & 90.7 & 90.7 & 90.7 & 90.5 & 90.7 & 90.7 & 90.7 & 90.1 & 90.3 \\
\hline $\begin{array}{l}\text { NY/150/ } \\
2000 / H 3 N 2 / \\
\text { HA/II }\end{array}$ & 93.1 & 93 & 93 & 93 & 92.4 & 92.5 & 92.5 & 92.5 & 92.4 & 92.5 \\
\hline $\begin{array}{l}\mathrm{CO} / 23619 / \\
99 / \mathrm{H} 3 \mathrm{~N} 2 / \\
\mathrm{HA} / \mathrm{II}\end{array}$ & 92.7 & 92.7 & 92.7 & 92.7 & 92.1 & 92.2 & 92.2 & 92.2 & 92.1 & 92.3 \\
\hline
\end{tabular}

genes originating from $\mathrm{A}(\mathrm{H} 1 \mathrm{~N} 1) \mathrm{pdm} 09$ virus. For the 2 Group 3 viruses, all internal genes were genetically related to $\mathrm{A}(\mathrm{H} 1 \mathrm{N1}) \mathrm{pdm09}$. Phylogenetic trees for the internal gene segments are presented in Additional file 2.

\section{HA1 amino acid analysis}

In order to determine whether the aa changes occurred in any of the previously identified antigenic sites A, B, C, D and $\mathrm{E}$ [7], we aligned the aa sequences of the 10 Ontario H3N2 viruses and compared them with the prototype cluster IV H3N2 virus [A/swine/Ontario/33853/2005/ (H3N2)]. Antigenic sites are shown in Figure 3. When compared to the known cluster IV H3N2 virus[A/swine/ Ontario/33853/2005/(H3N2)], the 4 Ontario H3N2 viruses classified in Group 1 had 8 aa changes within 4 antigenic sites, $\mathrm{A}(1), \mathrm{B}(3), \mathrm{C}(2)$ and $\mathrm{E}(2)$. The 4 Group 2 viruses had 8 aa changes within 3 antigenic sites, $A(3), B(3)$ and $C(2)$, and the 2 Group 3 viruses had 4 aa changes within 4 antigenic sites, $\mathrm{B}(1), \mathrm{D}(1)$ and $\mathrm{E}(2)$.

The Ontario H3N2 viruses from this study exhibited some unique changes at antigenic site B (Figure 3). Amino acids at position 155-160 (HNLDYK) related to prototype cluster IV virus were changed to YNLNYK (Group 1), YHLGHK (Group 2) and YHLNHK (Group 3). The receptor binding site (RBS) of the Ontario isolates examined in this study was conserved. The amino acids Y98, G134, S136, W153, H183, Y195, G225, and S227 were the same for the Ontario isolates and the prototype IV H3N2 virus (Figure 3). Identical amino acids of receptor binding pockets were also observed between isolates detected in this study and the turkey isolates reported previously and included in the analysis. The motif, N-X-T/S (X cannot be a proline) of the $\mathrm{N}$-glycosylation site also has been determined (Figure 3). Representative of cluster IV, the H3N2 [A/swine/Ontario/33853/2005/(H3N2)] virus had 7 potential glycosylation sites at positions $22,38,63,126$, 165, 246, and 285 (underlined in Figure 3). Seven potential glycosylation sites have been predicted for Group 1 at position $8,22,38,63,126,246$, and 285 . In this group one virus (A/swine/Ontario/114-13/2012(H3N2) had an additional potential glycosylation site at residue 165 . Six glycosylation sites have been predicted for Group 2 (22, $38,63,165,246$, and 285) and seven $(22,38,63,126,165$, 246, and 285) for Group 3 Ontario H3N2 viruses (underlined in Figure 3).

\section{Resistance-associated mutations}

Two classes of antiviral drugs are currently approved for prophylaxis and treatment of influenza A viruses in humans: adamantane derivatives (amantadine and rimantadine) and neuraminidase inhibitors (NAIs) (zanamivir and oseltamivir). To determine resistance-associated mutations to these two classes of drugs, we aligned and analyzed the aa sequences of the $\mathrm{M}$ and NA proteins of all 10 Ontario swH3N2 isolates (Figure 4). The most common mutation conferring resistance to adamantanes is an aa change from Ser to Asn at residue 31 (S31N) located in the transmembrane domain of the M2 protein. Sequence alignment analysis has shown that 6 of 10 Ontario swH3N2 isolates (Group 2, 3) do have the $\mathrm{S} 31 \mathrm{~N}$ mutation in the M2 protein, suggesting that Ontario swH3N2 isolates can be expected to offer resistance to amantadine and rimantadine (Figure 4). Besides the S31N mutation, additional substitutions have been traced. The R77Q substitution was observed only among 6 Ontario swH3N2 isolates with the $\mathrm{S} 31 \mathrm{~N}$ mutation. The remaining 4 isolates 
Table 2 Identity table of the full length of the NA gene of 10 Ontario H3N2 viruses isolated from swine in 2011-2012 and selected references viruses based on nucleotide sequence

\begin{tabular}{|c|c|c|c|c|c|c|c|c|c|c|}
\hline & $\begin{array}{l}\text { G1-A/sw/ } \\
\text { ON/114- } \\
\text { 13/12/ } \\
\text { H3N2/NA }\end{array}$ & $\begin{array}{l}\text { G1-A/sw/ } \\
\text { ON/115-2/ } \\
12 / \mathrm{H} 3 \mathrm{~N} 2 / \\
\mathrm{NA}\end{array}$ & $\begin{array}{l}\text { G1-A/sw/ } \\
\text { ON/118- } \\
38 / 12 / \\
\text { H3N2/NA }\end{array}$ & $\begin{array}{l}\text { G1-A/sw/ } \\
\text { ON/120- } \\
55 / 12 / \\
\text { H3N2/NA }\end{array}$ & $\begin{array}{l}\text { G2-A/sw/ } \\
\text { ON/104- } \\
\text { 25/12/ } \\
\text { H3N2/NA }\end{array}$ & $\begin{array}{l}\text { G2-A/sw/ } \\
\text { ON/204- } \\
76 / 12 / \\
\text { H3N2/NA }\end{array}$ & $\begin{array}{l}\text { G2-A/sw/ } \\
\text { ON/105- } \\
56 / 12 / \\
\text { H3N2/NA }\end{array}$ & $\begin{array}{l}\text { G2-A/sw/ } \\
\text { ON/107- } \\
22 / 12 / \\
\text { H3N2/NA }\end{array}$ & $\begin{array}{l}\text { G3-A/sw/ } \\
\text { ON/103- } \\
18 / 11 / \\
\text { H3N2/NA }\end{array}$ & $\begin{array}{l}\text { G3-A/sw/ } \\
\text { ON/11- } \\
105317 / \\
11 / \mathrm{H} 3 / \mathrm{NA}\end{array}$ \\
\hline $\begin{array}{l}\text { G1-A/sw/ } \\
\text { ON/114-13/ } \\
12 / H 3 N 2 / \\
\text { NA }\end{array}$ & 100 & 99.7 & 99.9 & 99.9 & 98.5 & 98.6 & 98.6 & 98.6 & 96 & 96 \\
\hline $\begin{array}{l}\text { G1-A/sw/ } \\
\text { ON/115-2/ } \\
12 / H 3 N 2 / \\
\text { NA }\end{array}$ & 99.7 & 100 & 99.9 & 99.9 & 98.8 & 98.9 & 98.9 & 98.9 & 96.1 & 96.2 \\
\hline $\begin{array}{l}\text { G1-A/sw/ } \\
\text { ON/118-38/ } \\
12 / H 3 N 2 / \\
\text { NA }\end{array}$ & 99.9 & 99.9 & 100 & 100 & 98.7 & 98.7 & 98.7 & 98.7 & 96 & 96 \\
\hline $\begin{array}{l}\text { G1-A/sw/ } \\
\text { ON/120-55/ } \\
12 / \mathrm{H} 3 \mathrm{~N} 2 / \\
\text { NA }\end{array}$ & 99.9 & 99.9 & 100 & 100 & 98.7 & 98.7 & 98.7 & 98.7 & 96 & 96 \\
\hline $\begin{array}{l}\text { G2-A/sw/ } \\
\text { ON/104-25/ } \\
12 / H 3 N 2 / \\
\text { NA }\end{array}$ & 98.5 & 98.8 & 98.7 & 98.7 & 100 & 99.9 & 99.9 & 99.9 & 96.3 & 96.4 \\
\hline $\begin{array}{l}\text { G2-A/sw/ } \\
\text { ON/204-76/ } \\
12 / H 3 N 2 / \\
\text { NA }\end{array}$ & 98.6 & 98.9 & 98.7 & 98.7 & 99.9 & 100 & 100 & 100 & 96.4 & 96.5 \\
\hline $\begin{array}{l}\text { G2-A/sw/ } \\
\text { ON/105-56/ } \\
12 / H 3 N 2 / \\
\text { NA }\end{array}$ & 98.6 & 98.9 & 98.7 & 98.7 & 99.9 & 100 & 100 & 100 & 96.4 & 96.5 \\
\hline $\begin{array}{l}\text { G2-A/sw/ } \\
\text { ON/107-22/ } \\
12 / H 3 N 2 / \\
\text { NA }\end{array}$ & 98.6 & 98.9 & 98.7 & 98.7 & 99.9 & 100 & 100 & 100 & 96.4 & 96.5 \\
\hline $\begin{array}{l}\text { G3-A/sw/ } \\
\text { ON/103-18/ } \\
11 / \mathrm{H} 3 \mathrm{~N} 2 / \\
\text { NA }\end{array}$ & 96 & 96.1 & 96 & 96 & 96.3 & 96.4 & 96.4 & 96.4 & 100 & 99.9 \\
\hline $\begin{array}{l}\text { G3-A/sw/ } \\
\text { ON/11- } \\
\text { 105317/11/ } \\
\text { H3N2/NA }\end{array}$ & 96 & 96.2 & 96 & 96 & 96.4 & 96.5 & 96.5 & 96.5 & 99.9 & 100 \\
\hline $\begin{array}{l}\text { TK/ON/ } \\
\text { 31232/05/ } \\
\text { H3N2/NA/IV }\end{array}$ & 97.5 & 97.7 & 97.5 & 97.5 & 98 & 98.1 & 98.1 & 98.1 & 98 & 98.1 \\
\hline $\begin{array}{l}\text { ON/33853/ } \\
05 / H 3 N 2 / \\
\text { NA/IV }\end{array}$ & 97.7 & 97.8 & 97.7 & 97.7 & 98.2 & 98.2 & 98.2 & 98.2 & 98 & 98.1 \\
\hline $\begin{array}{l}\text { QC/1698-4/ } \\
09 / \mathrm{H} 3 \mathrm{~N} 2 / \\
\text { NA/IV }\end{array}$ & 96.2 & 96.3 & 96.2 & 96.2 & 96.7 & 96.7 & 96.7 & 96.7 & 96.9 & 97 \\
\hline $\begin{array}{l}\text { QC/382/09/ } \\
\text { H3N2/NA/IV }\end{array}$ & 96.5 & 96.7 & 96.5 & 96.5 & 97 & 97.1 & 97.1 & 97.1 & 97.3 & 97.4 \\
\hline $\begin{array}{l}\text { QC/ } \\
1257777 / \\
2010 / H 3 N 2 / \\
\text { NA }\end{array}$ & 96.2 & 96.4 & 96.2 & 96.2 & 96.9 & 97 & 97 & 97 & 96.9 & 97 \\
\hline
\end{tabular}


Table 2 Identity table of the full length of the NA gene of 10 Ontario H3N2 viruses isolated from swine in 2011-2012 and selected references viruses based on nucleotide sequence (Continued)

\begin{tabular}{|c|c|c|c|c|c|c|c|c|c|c|}
\hline $\begin{array}{l}\text { QC/ } \\
1267566 / \\
2010 / H 3 N 2 / \\
\text { NA }\end{array}$ & 95.6 & 95.7 & 95.6 & 95.6 & 96.1 & 96.2 & 96.2 & 96.2 & 97 & 97 \\
\hline $\begin{array}{l}\text { QC/ } \\
1267568 / \\
2010 / H 3 N 2 / \\
\text { NA }\end{array}$ & 95.3 & 95.5 & 95.3 & 95.3 & 95.8 & 95.9 & 95.9 & 95.9 & 96.2 & 96.2 \\
\hline $\begin{array}{l}\text { TK/ON/ } \\
\text { FAV9/2011/ } \\
\text { H3N2/NA }\end{array}$ & 98.3 & 98.6 & 98.4 & 98.4 & 99.4 & 99.4 & 99.4 & 99.4 & 96.1 & 96.2 \\
\hline $\begin{array}{l}\text { TK/ON/ } \\
\text { FAV10/ } \\
2011 / \mathrm{H} 3 \mathrm{~N} 2 / \\
\text { NA }\end{array}$ & 98.4 & 98.7 & 98.6 & 98.6 & 99.5 & 99.6 & 99.6 & 99.6 & 96.3 & 96.3 \\
\hline $\begin{array}{l}\text { QC/ } \\
1262080 / \\
2010 / H 3 N 2 / \\
\text { NA }\end{array}$ & 95.5 & 95.6 & 95.5 & 95.5 & 96 & 96 & 96 & 96 & 95.5 & 95.6 \\
\hline $\begin{array}{l}\mathrm{QC} / \\
1257774 / \\
2010 / \mathrm{H} 3 \mathrm{~N} 2 / \\
\text { NA }\end{array}$ & 95.3 & 95.5 & 95.3 & 95.3 & 95.8 & 95.9 & 95.9 & 95.9 & 95.7 & 95.7 \\
\hline $\begin{array}{l}\text { QC/ } \\
1265553 / \\
2010 / H 3 N 2 / \\
\text { NA }\end{array}$ & 95.4 & 95.5 & 95.4 & 95.4 & 95.9 & 96 & 96 & 96 & 95.5 & 95.5 \\
\hline $\begin{array}{l}\mathrm{BC} / 28103 / \\
05 / \mathrm{H} 3 \mathrm{~N} 2 / \\
\mathrm{NA} / \mathrm{IV}\end{array}$ & 97.5 & 97.7 & 97.5 & 97.5 & 98 & 98.1 & 98.1 & 98.1 & 97.9 & 97.9 \\
\hline $\begin{array}{l}\text { MN/12707/ } \\
\text { 05/H3N2/ } \\
\text { NA/IV }\end{array}$ & 96.9 & 97 & 96.9 & 96.9 & 97.4 & 97.4 & 97.4 & 97.4 & 97.2 & 97.3 \\
\hline $\begin{array}{l}\text { AB/14722/ } \\
05 / H 3 N 2 / \\
\text { NA/IV }\end{array}$ & 96.7 & 96.8 & 96.7 & 96.7 & 97.2 & 97.2 & 97.2 & 97.2 & 97 & 97.1 \\
\hline $\begin{array}{l}\text { OK/18717/ } \\
\text { 99/H3N2/ } \\
\text { NA/III }\end{array}$ & 93.8 & 93.9 & 93.8 & 93.8 & 94.1 & 94.2 & 94.2 & 94.2 & 94.2 & 94.3 \\
\hline $\begin{array}{l}\text { IL/21587/ } \\
\text { 99/H3N2/ } \\
\text { NA/III }\end{array}$ & 93.7 & 93.8 & 93.7 & 93.7 & 94 & 94.1 & 94.1 & 94.1 & 94.1 & 94.2 \\
\hline $\begin{array}{l}\text { WI/14094/ } \\
99 / H 3 N 2 / \\
\text { NA/III }\end{array}$ & 93.7 & 93.9 & 93.7 & 93.7 & 94.1 & 94.1 & 94.1 & 94.1 & 94.1 & 94.2 \\
\hline $\begin{array}{l}\text { OK/18089/ } \\
99 / H 3 N 2 / \\
\text { NA/III }\end{array}$ & 93.8 & 93.9 & 93.8 & 93.8 & 94.1 & 94.2 & 94.2 & 94.2 & 94.2 & 94.3 \\
\hline $\begin{array}{l}\mathrm{NC} / 16497 / \\
99 / \mathrm{H} 3 \mathrm{~N} 2 / \\
\mathrm{NA} / \mathrm{l}\end{array}$ & 93.5 & 93.8 & 93.7 & 93.7 & 94 & 94.1 & 94.1 & 94.1 & 93.9 & 94 \\
\hline $\begin{array}{l}\text { TX/4199-2/ } \\
98\end{array}$ & 93.7 & 93.9 & 93.7 & 93.7 & 94.1 & 94.2 & 94.2 & 94.2 & 94.2 & 94.2 \\
\hline $\begin{array}{l}\text { IA/8548-1/ } \\
98 / H 3 N 2 / \\
\text { NA/I }\end{array}$ & 93.4 & 93.6 & 93.4 & 93.4 & 93.8 & 93.9 & 93.9 & 93.9 & 93.9 & 94 \\
\hline
\end{tabular}


Table 2 Identity table of the full length of the NA gene of 10 Ontario H3N2 viruses isolated from swine in 2011-2012 and selected references viruses based on nucleotide sequence (Continued)

\begin{tabular}{|c|c|c|c|c|c|c|c|c|c|c|}
\hline $\begin{array}{l}\mathrm{CO} / 23619 / \\
99 / \mathrm{H} 3 \mathrm{~N} 2 / \\
\mathrm{NA} / \mathrm{II}\end{array}$ & 93.7 & 93.9 & 93.7 & 93.7 & 94.2 & 94.3 & 94.3 & 94.3 & 94.3 & 94.4 \\
\hline $\begin{array}{l}\text { NY/150/ } \\
2000 / H 3 N 2 / \\
\text { NA/II }\end{array}$ & 94.5 & 94.7 & 94.5 & 94.5 & 95 & 95.1 & 95.1 & 95.1 & 95 & 95.1 \\
\hline
\end{tabular}

without S31N mutation exhibited a V27I substitution (Figure 4).

Resistance-associated mutations within the neuraminidase E119V, R292K and N294S, responsible for reduced susceptibility to oseltamivir, were not detected in any of the Ontario swH3N2 sequences (data not shown).

\section{Signature residues}

Influenza virus segment 2 encodes 3 proteins, PB1, PB1F2 encoded by an alternative open reading frame (ORF) of segment 2, and PB1 N40 N-terminally truncated version of the PB1 protein. Phylogenetic analysis of 32 PB1 aa sequences showed that PB1-F2 of all 10 Ontario H3N2 viruses belongs to lineage D (Additional file 3).

Eight of 10 Ontario H3N2 viruses (Figure 5) had complete 90 aa PB1-F2 fragments (long bar). The remaining 2 viruses (A/sw/Ontario/103-18/2011(H3N2)) and (A/sw/Ontario/11-105317/2011(H3N2)) from Group 3 expressed a truncated form of PB1-F2 containing 11 amino acids (short bar), lacking the mitochondrial targeting sequence (MTS) located in the C-terminal region. Further sequence analysis of Ontario H3N2 PB1-F2 proteins revealed the absence of the N66S mutation which was previously reported in the H1N1, 1918 PB1-F2 and pathogenic avian $\mathrm{H} 5 \mathrm{~N} 1$ viruses and found to be associated with a gain in virulence. In addition to N66S, further amino acids T51, V56 and E87 might also enhance pathogenicity. As can be seen from Figure 5 the aa sequences of Ontario H3N2 PB1-F2 all contain V56, but substitutions T51M and $\mathrm{E} 87 \mathrm{G}$, previously associated with lower pathogenicity, have also been determined [8].

Further, we assessed PB2 signature amino acids, which greatly influences host range. The PB2 key signature aa at position 627 is an important determinant of virulence and host specificity [9-11]. This analysis demonstrated the absence of a PB2 E627K mutation, but revealed 59091SR and 271A mutations in all Ontario H3N2 viruses (data not shown). The H1N1 2009 pandemic viruses also

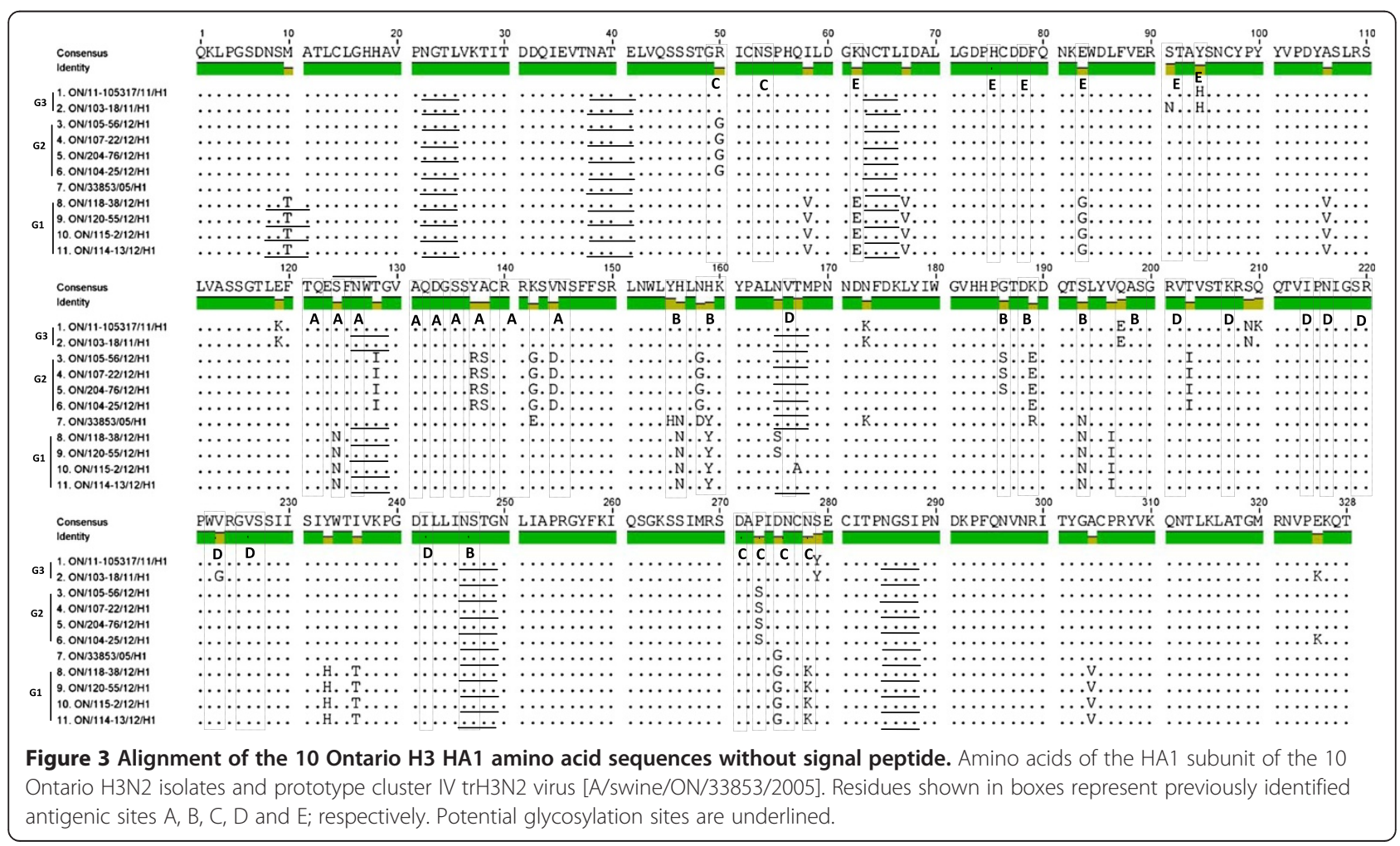




\begin{tabular}{|c|c|}
\hline & V27I \\
\hline G1-A/sw/ON/114-13/12/H3N2/M2 & MSLLTEVETPIRNGWECKCNDSSDPLID DASI I GILHLILWILDRLFFKCIYRRFKYGLQRGPSTEGVPESMREEYRQKQQSAVDVDDGHFVNIVLE \\
\hline G1-A/sw/ON/115-2/12/H3N2/M2 & MSLITEVETPIRNGWECKCNDSSDPL'I DAASI I GI LHLILWILDRLFFKCIYRRFKYGLQRGPSTEGVPESMREEYRQKQQSAVDVDDGHFVNIVLE \\
\hline G1-A/sw/ON/118-38/12/H3N2/M2 & MSLLTEVETPIRNGWECKCNDSSDPLII DAASI I GILHLILWILDRLFFKCIYRRFKYGLQRGPSTEGVPESMREEYRQKQQSAVDVDDGHFVNIVLE \\
\hline G1-A/sw/ON/120-55/12/H3N2/M2 & $\begin{array}{l}\text { MSLLTEVETPIRNGWECKCNDSSDPLII \$AASI I GI LHLI LWILDRLFFKCIYRRFKYGLQRGPSTEGVPESMREEYRQKQQSAVDVDDGHFVNIVLE } \\
\mathbf{S 3 1 N}\end{array}$ \\
\hline G2-A/sw/ON/204-76/12/H3N2/M2 & MSLITEVETPTRREWECRCSDSSDPLVIAANN L I GILHLILWITDRLFFKCIYRRFKYGLKRGPSTEGVPESMREEY SpEQQSAVDVDDGHFVNIELE \\
\hline G2-A/sw/ON/104-25/12/H3N2/M2 & MSLITEVETPTRREWECRCSDSSDPLVIAAłN L I GILHLILWITDRLFFKCIYRRFKYGLKRGPSTEGVPESMREEY Y \\
\hline G2-A/sw/ON/105-56/12/H3N2/M2 & 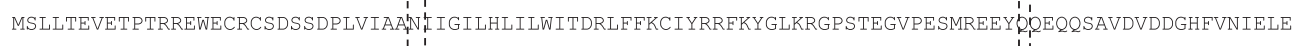 \\
\hline G2-A/sw/ON/107-22/12/H3N2/M2 & 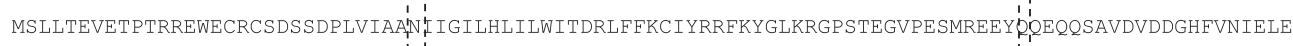 \\
\hline G3-A/sw/ON/103-18/11/H3N2/M2 & MSLLTEVETPTRSEWECRCSDSSDPLVIAATN I I GILHLILWITDRLFFKCIYRRFKYGLKRGPSTEGVPESMREEY \\
\hline \multirow[t]{2}{*}{ G3-A/sw/ON/11-105317/11/H3N2/M2 } & MSLLTEVETPTRSEWECRCSDSSDPLVIAANIIGILHLILWITDRLFFCIYRRFKYGLKRGPSTEGVPESMREEY Y QEQQSAVDVDDGHFVNIELE \\
\hline & M2 TM (aа24-46) \\
\hline 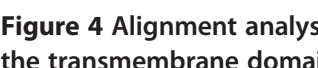 & of M2 sequences of 10 Ontario swH3N2 isolates showing amino acid substitution S31 \\
\hline
\end{tabular}

\begin{tabular}{|c|c|c|c|c|c|c|c|c|c|c|}
\hline & & 10 & 20 & 30 & 40 & 50 & 60 & 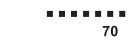 & 80 & 90 \\
\hline \multirow[t]{3}{*}{ Lineage $A$} & A/Brevig Mission/1/1918(H1N1) & MGQEQDTPWI & LSTGHISTQK & REDGQQTPRL & EHHNSTRLMD & HCQKTMNQVV & MPKQIVYWKQ & WLSLRSPTPV & SLKTRVLKRW & RLFSKHEWTS \\
\hline & A/Puerto Rico/8/1934(H1N1) & MGQEQDTPWI & LSTGHISTQK & REDGQQTPKL & EHRNSTRLMG & HCQKTMNQVV & MPKQIVYWRR & WLSLRNPILV & FLKTRVLKRW & RLFSKHE`TS \\
\hline & A/swine/Memphis/1/1990(H1N1) & MGQEQDTLWI & L*TEHISTQK & EENGQ*TPKL & VRHN*IQLMD & RCLRTMNQLV & MPKQTAF*KQ & WRFSRNPILA & SLKTHVLKRW & RLFSKHGWTN \\
\hline Lineage $B$ & A/turkey/Ontario/7732/1966(H5N9) & MEQEQDTPWI & QSTGHINIQK & RENGQQTLKL & EHHNSIQLMD & HCPKTMNRAV & MLKQTVCWRQ & WLFLRSPTPV & SLKTHVLRRW & KSFSKHGWTN \\
\hline Lineage $C$ & A/chicken/Rostock/45/1934(H7N1) & MEREQGTPWT & QLIGHTNIQK & RGNGQQTRRL & EHPNSTQLMD & HYLRTMSQVD & MHRQTASWRQ & WLSLKSLTPE & SLKTHVLRRW & KLFSKQE*TN \\
\hline \multirow[t]{14}{*}{ Lineage $D$} & A/Hong Kong/1/68(H3N2 & MEQEQDTPWT & QSTEHINIQK & KGSGQQTRKL & ERPNLTQLMD & HYLRIMSQVD & MHKQTVSWKQ & WLSLKNPTQG & SLKTRVLKRW & KLFNKQGWTD \\
\hline & A/Panama/2007/1999(H3N2) & MEQEQGTPWT & QSTEHTNIQK & RGSGRQIQKL & GHPNSTQLMD & HYLRIMSQVD & MHKQTVSWRL & WPSLKNPTQG & SLRTHALKQW & KSFNKQGWTN \\
\hline & A/swine/Minnesota/66853/2006(H3N2) & MEQEQDTPWT & QSTEHTNIQK & KGNGRQTQRL & GHPSSTRLMD & HYLKIMNQVD & MHKQTVSWRP & WLSLKNPTQG & YLRIHALKQW & KLSNKQGWIN \\
\hline & A/Hamburg/4/2009(H1N1) & MEQEQDTPWT & Q*TEHTNTQK & RESGRQTQRL & VHPSSTRLMD & HYLRIMNQVG & MHKQTVF*RL & WLSLKNPTQE & YLRIHALKQW & KLFNKQG*IN \\
\hline & G2-A/swine/ON/104-25/2012/H3N2 & MEQEQDTPWT & QSTEHTNIQK & KGNGRQTQRL & GHPSSTRLMD & HYLKIMNQVD & MHKQTVSWRP & WLSLKNPTQG & YLRIHALKQW & KLSNKQGWIN \\
\hline & G2-A/swine/Ontario/204-76/2012/H3N2 & MEQEQDTPWT & QSTEHTNIQK & KGNGRQTQRL & GHPSSTRLMD & HYLKIMNQVD & MHKQTVSWRP & WLSLKNPTQG & YLRIHALKQW & KLSNKQGWIN \\
\hline & G2-A/swine/Ontario/105-56/2012/H3N2 & MEQEQDTPWT & QSTEHTNIQK & KGNGRQTQRL & GHPSSTRLMD & HYLKIMNQVD & MHKQTVSWRP & WLSLKNPTQG & YLRIHALKQW & KLSNKQGWIN \\
\hline & G2-A/swine/Ontario/107-22/2012/H3N2/HA & MEQEQDTPWT & QSTEHTNIQK & KGNGRQTQRL & GHPSSTRLMD & HYLKIMNQVD & MHKQTVSWRP & WLSLKNPTQG & YLRIHALKQW & KLSNKQGWIN \\
\hline & G1-A/swine/Ontario/114-13/2012/H3N2/HA & MEQEQDTPWT & QSTEHTNIQK & KGNGRQTQRL & GHPSSTRLMD & HYLKIMNQVD & MHKQTVSWRP & WLSLKNPTQG & YLRIHALKQW & KLSNKQGWIN \\
\hline & G1-A/swine/Ontario/115-2/2012/H3N2/HA & MEQEQDTPWT & QSTEHTNIQK & KGNGRQTQRL & GHPSSTRLMD & HYLKIMNQVD & MHKQTVSWRP & WLSLKNPTQG & YLRIHALKQW & KLSNKQGWIN \\
\hline & G1-A/swine/Ontario/118-38/2012/H3N2/HA & MEQEQDTPWT & QSTEHTNIQK & KGNGRQTQRL & GHPSSTRLMD & HYLKIMNQVD & MHKQTVSWRP & WLSLKNPTQG & YLRIHALKQW & KLSNKQGWIN \\
\hline & G1-A/swine/Ontario/120-55/2012/H3N2/HA & MEQEQDTPWT & QSTEHTNIQK & KGNGRQTQRL & GHPSSTRLMD & HYLKIMNQVD & MHKQTVSWRP & WLSLKNPTQG & YLRIHALKQW & KLSNKQGWIN \\
\hline & G3-A/swine/Ontario/103-18/2011/H3N2 & MEQEQDTPWT & $Q^{\star}$ TEHTNTQK & RESGRQTQRL & VHPSSTRLMD & HYLRIMNQVG & MHKQTVFRL & WLSLKNPTQE & YLRIHALKQW & KLFNKQG*IN \\
\hline & G3-A/swine/Ontario/11-105317/2011/H3N2 & MEQEQDTPWT & Q*TEHTNTQK & RESGRQTQRL & VHPSSTRLMD & HYLRIMNQVG & MHKQTVFRL & WLSLKNPTQE & YLRIHALKQW & KLFNKQG ${ }^{*} I N$ \\
\hline \multirow[t]{4}{*}{ Lineage $E$} & A/duck/Czech Republic/1/1956(H4N6) & MEQEQDTPWT & QSTEHINTQK & GGSGQQTRKL & ERPNSTQLMD & HYLRTMNQVD & MHKQTASWKQ & WLSLKDPTQE & SLKIHVLKRW & KLFNKQEWTN \\
\hline & A/Leningrad/134/57(H2N2) & MEQEQDTPWT & QSTEHINIQK & RGSGQQTRKL & ERPNLTQLMD & HYLRTMNQVD & MHKQTASWKQ & WLSLKNPTQE & SLKTRVLKRW & RLFNKQEWTN \\
\hline & A/Singapore/1/1957(H2N2) & MEQEQDTPWT & QSTEHINIQK & RGSGQQTRKL & ERPNLTQLMD & HYLRTMNQVD & MHKQTASWKQ & WLSLKNPTRE & SLKTRVLKRW & KLFNKQEWTN \\
\hline & A/quail/ttaly/1117/1965(H10N8) & MEQEQDTPWT & QSTEHINTQK & KGNGQQIRKL & ERPNSTQLMD & HYLRAMSQVG & MHRQTASWKP & WPSLKNPTQE & SLKTRVLKRW & KLFNKQGWTR \\
\hline \multirow[t]{2}{*}{ Lineage $F$} & A/swine/Saskatchewan/18789/02(H1N1) & MERERDTPWT & QSTGHINTQK & RGNGQQTQRL & EHPNLTRLTD & HYLRIMNQVD & MHKQTASWKQ & WLSLKNPTQE & SLKTRVLKRW & KLFNKQEWTS \\
\hline & A/American black duck/NB/2538/2007(H7N3) & MEQEQDTPWT & RLTEHINIQK & RENGQQTQRL & GHPNLTQLTD & HCLRTMSQVD & MHKRTVSLKQ & WLSLKSPTQE & SLKTRVLKRW & KLFNKQEWTS \\
\hline \multirow[t]{3}{*}{ Lineage $G$} & A/swine/Spain/39139/2002(H3N2) & MEQGQDTPWT & RSTGHINIQK & KENGQQTQRL & EHPSLTR*MG & HYLRTTNQAD & MHRQTVSWKQ & WLSLRSPTQE & YLKTHVLRRL & RLYNKOEWTN \\
\hline & A/swine/Bakum/1832/2000(H1N2) & MEQGQDTPWT & RSTEHINIQK & GEDGQQTQKL & EHPSLTRLMD & HYLRTMSQAG & MLKQTVSWRQ & WLSLRNPTQG & YLKTRVLKR* & RLPNKQEWTN \\
\hline & A/swine/France/WVL8/1992(H1N1) & MEQGQDTPWT & QSTEHINIQK & RESGQQTQRL & EHPSLTRLMD & HYPRTMNQAD & MHKQIVSWRQ & WLSLKNPTQG & YLKTHVLRRW & RLFSKQEะTN \\
\hline \multirow[t]{2}{*}{ Lineage $\mathrm{H}$} & A/duck/New Zealand/78/1984(H9N2) & MEQEQGTPWT & QSTGHINTQK & RGNGQQTQKL & EHPNSIQLMG & HYLKTTNQVG & THKQTVSWKa & WLSLKNPTQG & SLKTRVLKRW & KLFNRQGLTS \\
\hline & A/duck/NZL/41/1984(H1N2) & MEQEQDTPWT & QSTGHINTQK & RGNGQQTQKL & EHPNSIQLMG & HYLKTTNQVG & MHKQTASWKQ & WLSLKNPTQG & SLKTRVLKRW & KLFNRQGLTS \\
\hline \multirow[t]{2}{*}{ Lineage I } & A/swine/Anhui/01/2006(H3N8) & MEQGQDTPWI & LSTEHINTQK & RGNGQQTLRL & EHHNLIQSMD & RFLKTMNQAVT & TPKQIVYWKQ & WLSLKNPIPG & SLKIRVLKRW & RWFNRQEWTN \\
\hline & Alequine/Austria/421/1992(H3N8) & MEQGQDTPWI & LSTEHINTQK & RGNGQQTLRL & EHHNLIQSMD & RFLKTMNQAGT & TPKQIVYWKQ & WLSLKNPIPG & SLKIRVLKRW & RWFNRQEWTN \\
\hline \multicolumn{11}{|c|}{$\begin{array}{l}\text { Figure } 5 \text { Presentation of PB1-F2 variants of mammalian and avian influenza A viruses. Nine PB1 lineages from A to I have been described } \\
\text { and some selected sequences within these lineages are presented. Lines at the top of the Figure represent amino acids of the predicted helical } \\
\text { region (black) and the putative mitochondrial targeting sequence (dashed). Amino acids that are considered to enhance viral pathogenesis are } \\
\text { marked in grey. The first stop codon has been shown by asterisk and following stop codons are indicated by subsequent asterisk. }\end{array}$} \\
\hline
\end{tabular}


had PB2 627E and carried PB2 590-91SR and 271A mutations. Such a distribution of mutations might compensate for PB2 627E and adapt the viruses for efficient replication in mammals.

Signature residues of the PA protein have also been assessed and showed the absence of PA 100A that is present in human influenza $\mathrm{A}$ viruses that caused the pandemic of $1977 \mathrm{~A}(\mathrm{H} 1 \mathrm{~N} 1), 1968 \mathrm{~A}(\mathrm{H} 3 \mathrm{~N} 2)$, and $1957 \mathrm{~A}$ (H2N2). Instead of 100A, Ontario swH3N2 isolates had PA $100 \mathrm{~V}$. Additional mutations, PA 356R and $409 \mathrm{~N}$ associated with past influenza pandemics, including 2009 (H1N1), 1977 (H1N1), 1968 (H3N2) and 1997 (H5N1), respectively, have also been detected in Ontario swH3N2 isolates (data not shown).

Close attention has been paid to the NP protein as a part of the helical genomic ribonucleoprotein complex with an important role in viral RNA replication and crossspecies transmission. The NP protein with isoleucine at position NP 100, associated with increased human-tohuman transmissibility, found in both 2009 and 1918 H1N1 pandemic viruses, has not been detected in Ontario swH3N2 isolates. Instead of an isoleucine residue, glycine was detected (data not shown).

\section{Discussion}

In this report we describe the genetic and predicted antigenic characterization of 10 Ontario H3N2 viruses isolated from swine in 2011 and 2012. On the basis of these results it can be concluded that at least three different groups of H3N2 viruses were circulating in Ontario swine during that time.

Group 1 represents viruses similar to the original trH3N2 viruses with gene segments of avian (PB2, PA), human (PB1, HA, NA) and classical swine (NP, M, NS) influenza virus origin. These 4 trH3N2 genotype viruses, with a mix of classical avian, human and swine virus lineages, emerged in 1998 and rapidly spread throughout the US swine population [12,13]. In 2005, the spread of these viruses to Canada were reported in Canadian swine, turkey, and mink populations [3,4]. Group 2 represents viruses with $\mathrm{HA}, \mathrm{NA}, \mathrm{PB} 1$, and NS genes closely related to trH3N2 viruses. The remaining internal genes are genetically related to $\mathrm{A}(\mathrm{H} 1 \mathrm{~N} 1) \mathrm{pdm} 09$ virus. These 4 Ontario H3N2 viruses exhibited the same gene assemblage as those found in trH3N2 influenza viruses isolated from breeder turkey flocks in Southern Ontario during 2011 (FAV-009 and FAV-010). Group 3 represents viruses with HA and NA genes closely related to trH3N2 viruses, with all internal genes genetically related to $\mathrm{A}(\mathrm{H} 1 \mathrm{~N} 1) \mathrm{pdm} 09$. Although structurally the same gene assemblage has been observed as in the recent Quebec isolates [14], more detailed analysis showed that some gene segments in this group of 2 viruses were most similar to the Ontario H3N2 historical isolates from 2005, suggesting that these viruses likely were of Ontario origin, and not introduced from Quebec, despite frequent swine movement between the two Canadian provinces. In 2009, a previously unrecognized H1N1 swine-origin subtype comprising a gene combination of North American and Eurasian IAV-S was isolated from humans in the US and Mexico [15]. This virus spread globally as the first pandemic of the 21th century. Shortly after the virus was first isolated from humans in the USA and Mexico, human-to-animal transmission was reported in Canada by Howden et al. [16] and Berhane et al. [17]. Virus isolates from Canadian pigs were phylogenetically similar to $\mathrm{A}(\mathrm{H} 1 \mathrm{~N} 1) \mathrm{pdm} 09$ virus $[16,18]$, which is often detected in the Canadian swine population [19]. The characterization of the Ontario H3N2 viruses clearly indicates reassortment of gene segments between the North American swine trH3N2 from Cluster IV and the $A(H 1 N 1) p d m 09$. Structurally similar viruses have been observed on multiple occasions since A(H1N1)pdm09 emerged in 2009 [4,20]. More recently, Kitikon et al. [21] reported at least ten unique groups of $\mathrm{H} 3 \mathrm{~N} 2$ reassortants based on $\mathrm{H} 3 \mathrm{~N} 2 / \mathrm{H} 1 \mathrm{~N} 1 \mathrm{pdm}(09)$ gene combination. Findings in this study have implications for animal and public health, because H3N2 viruses with the matrix gene from H1N1pdm09 were implicated in influenza outbreaks in people attending agricultural fairs in US in 2012 [22-24], showed efficient droplet transmission in experimental studies on ferrets [25], and increased person-to-person transmissibility in modelling studies based on outbreak data in people [26].

The percentage of aa identity of HA1 between the prototype Cluster IV trH3N2 virus [A/swine/Ontario/33853/ 2005/(H3N2)] and 10 Ontario H3N2 viruses ranged from $94.5 \%$ to $95.7 \%$. We detected 8 aa changes at 4 antigenic sites (A, B, C and E) for Group 1 viruses, 8 aa changes at 3 antigenic sites (A, B and C) for Group 2, and 4 aa changes at 3 antigenic sites (B, D and E) for Group 3. According to Wilson and Cox [27], a drift variant with $\geq 4$ aa changes at $\geq 2$ of 5 antigenic sites could be of epidemiologic importance. Additionally, Wood et al. [28] and Kodihalli et al. [29] have shown that 1 to 3 aa changes in the HA1 of H1N1 and $\mathrm{H} 3 \mathrm{~N} 2$ viruses can reduce cross reactivity and efficacy of inactivated vaccine. This leaves open the possibility that several variants of $\mathrm{H} 3 \mathrm{~N} 2$ could co-circulate in the same population. However, more detailed serological studies and field studies should be done to better appreciate the potential for such co-circulation or the frequency with which this occurs.

Although, the Ontario H3N2 viruses from this study shared 5 potential N-linked glycosylation sites at positions $22,38,63,126$, and 285 with the closely related cluster IV A/swine/Ontario/33853/2005/(H3N2) virus, Group 2 (4 viruses) lacked a potential glycosylation site at position 126, Group 1 (4 viruses) had an additional 
potential glycosylation site at position 8 , and 3 viruses in Group 1 lacked a glycosylation site at position 165 . The existence of $\mathrm{N}$-glycosylation is essential for viral membrane glycoproteins. The $\mathrm{N}$-glycans encode required information for folding, maturation, transport or degradation of proteins [30]. Glycosylation seems to be one mechanism by which a virus masks its epitopes and escapes neutralization by the host antibodies [31,32]. However, the study of Vigerust et al. [33] linked increased glycosylation of influenza viruses with decreased virulence.

The M2 is the target of the anti-influenza drugs amantadine and rimantadine. Resistance-associated mutation to adamantanes S31N has been detected in 6 Ontario H3N2 viruses indicating resistance to amantadine and rimantadine. According to our findings at least 6 Ontario swH3N2 viruses out of 10 evaluated can be expected to offer resistance to amantadine and rimantadine.

In conclusion, this study showed that at least 3 different variants of $\mathrm{H} 3 \mathrm{~N} 2$ viruses circulated in Ontario swine in 2011 and 2012. Group 1 viruses are most closely related to trH3N2 viruses from 2005 [3]. Group 2 viruses are closely related to $\mathrm{H} 3 \mathrm{~N} 2$ viruses that were previously reported in Ontario turkeys [6]. It is important to note that there were no clear epidemiological linkages among swine and turkey farms with detected viruses, although no detailed disease investigations were performed as a part of this study. Group 3 viruses are most closely related to swH3N2/A(H1N1)pdm09 reassortant viruses from swine in the province of Quebec, but are likely of Ontario origin. According to amino acid sequence analysis of the M2 protein, Ontario H3N2 viruses can be expected to offer resistance to adamantane derivatives, but not to neuraminidase inhibitors.

\section{Materials and methods Study populations}

Invitation for participation in the study was distributed through the Ontario Association of Swine Veterinarians' email distribution list. Inclusion criterion for these herds was the current or recent presence of clinical signs suggestive of infection with influenza A virus as indicated by a herd veterinarian. Twenty-one Ontario swine herds were sampled between November 2011 and May 2013, with the majority of 18 herds sampled in 2012. Groups and animals showing clinical signs suggestive of influenza were targeted for sampling. Dacron-tipped nasal swabs were collected from both nostrils of individual animals, deposited in PBS, and transported to a virology laboratory (Department of Pathobiology, University of Guelph). Nasal swabs were either tested within $24 \mathrm{~h}$ or frozen at $-80^{\circ} \mathrm{C}$ before being inoculated in MDCK cells. The originally planned sample size was 100 per herd, sufficient to detect a prevalence of infection between $2 \%$ and $3 \%$. However, actual sample size varied depending on the prevalence of clinical signs and resources available for sampling.

In twelve of those 21 herds an additional 30 nasal swabs were collected from individual animals with clinical signs indicative of influenza. These samples were transported in $2 \mathrm{ml}$ of VTM and submitted to the Animal Health Laboratory (AHL, University of Guelph, ON, Canada). The samples were then pooled 3:1 and tested with a rtRT-PCR directed to the matrix protein of influenza A viruses.

Eleven viruses from 10 herds that tested positive for H3N2 influenza virus were included in the full genome sequencing study. The viruses were selected purposively so that only one farm per production system was included. For 9 herds, one randomly selected virus was included; and for one herd, 2 randomly selected viruses were included.

\section{Samples submitted to the virology laboratory of the department of pathobiology}

Swab samples were added to MDCK cells as described elsewhere [34]. Virus isolates were identified by a hemagglutination (HA) assay, as described elsewhere [34].

Viral RNA was extracted using the RNeasy Mini Kit according to the manufacturer's instructions (Qiagen Inc., Valencia, CA, USA). Briefly, the cDNA was synthesized with Random Primer (Invitrogen, Canada Inc., Burlington, ON, Canada) and by SuperScript ${ }^{\mathrm{Tm}}$ II Reverse Transcriptase, Invitrogen, Canada Inc Burlington, ON, Canada) according to the manufacturer's instruction. The subtype was determined by reverse transcription (RT-PCR) and further characterized by full genome sequencing using 454 sequencing technology. The RT-PCR subtyping was performed on the harvested virus infected cell culture supernatant, as identified by CPE and HA. Primer pairs utilized in study for subtype determination were: HA gene (subtype H1Forw 5'-GTTCTGCTATATACATTTGC-3', H1Rev 5'-GACCCATTAGAGCACATC-3' or H3Forw 5'-GGA-AGT-GAC-AAC-AGC-AT-3', H3Rev (5'-AGCTGA-ACA-CCT-TTG-ATC-T-3') 5' - and NA gene (subtype N1Forw 5' - TAACATCAGCAACATCAACT-3', N1Rev GTCGCCCTCTGATTAGTT-3' or N2 Forw 5'GGCTCTGTTTCTCTCATC-3', N Rev 5'- CAAGTCC TGAGCATACATA-3'). The PCR was performed with 35 cycles of $94^{\circ} \mathrm{C}$ for $15 \mathrm{~s}, 54^{\circ} \mathrm{C}$ for $30 \mathrm{~s}$, and $72^{\circ} \mathrm{C}$ for $60 \mathrm{~s}$. The results of the RT-PCR assay designated the influenza virus type $A$ virus to be either subtype $\mathrm{H} 1 \mathrm{~N} 1$ or $\mathrm{H} 3 \mathrm{~N} 2$.

\section{Samples submitted to the AHL}

Viral RNA was extracted on an automated platform using the MagMAX ${ }^{\mathrm{TM}}$ Express-96 instrument and MagMAX ${ }^{\mathrm{ms}} 96$ Viral RNA Isolation kit (cat\#AM1836), according to the manufacturer's instructions. To identify swine influenza A viruses a rtRT-PCR was used with matrix primers 
redesigned by Dr J. Pasick (CFIA, Winnipeg, MB,CA), enhanced for the detection of $\mathrm{A}(\mathrm{H} 1 \mathrm{~N} 1) \mathrm{pdm} 09$, using SIV Forward Primer - 5'd AGA TGA GTC YTC TAA CCG AGG TCG 3'; SIV Reverse Primer - 5'd TGC AAA RAC AYY TTC MAG TCT CTG 3'. An AIV M + 64 Probe -5' FAM-TCA GGC CCC CTC AAA GCC GA-BHQ-1 3' designed by Spackman et al. [35] was used. The probe was purchased from Biosearch Technologies as Reference \# SS113269-29. These were used in combination with the Ambion AgPath-ID one-step RT-PCR Kit Cat \#AM1005, according to the manufacturer's instructions.

If the matrix rtRT-PCR was positive, then the specimen was processed for virus isolation using MDCK cells and rtRT-PCR subtyping was performed on the harvested virus infected cell culture supernatant, as identified by CPE and HA using chicken, turkey or guinea pig red blood cells. Where large concentrations of virus were present in clinical samples, as evidenced by low CT values in the matrix rtRT-PCR, subtyping was attempted directly from clinical samples. However in the majority of cases clinical samples did not have high enough levels of viral RNA for successful subtyping and cell culture supernatant was used.

The first step for subtyping was initial production of complementary DNA (cDNA) from RNA extracted from MDCK or allantoic fluid culture isolates using random hexamer priming and MuLV reverse transcriptase. During the second step the cDNA was amplified using single primer pairs to type the isolate as H1N1 or H3N2. Primer pairs were complementary to the HA gene (subtype H1 -612 F5'-GGT GTG ACG GCA GCA TGC CCT-3'; H1 612 R 5'-AGC AAT GGC TCC AAA CAG ACC-3' or H3 722 F 5'-CARATTGARGTGACHAATGC-3'; H3 722 R $5^{\prime}$-GGTGCATCTGAYCTCATTA-3') and the NA gene (subtype N1 533 F 5'-CTT CCT ATC CAA ACA CCA TT-3'; N1 533 R5'-TTG CTT GGT CAG CAA GTG-3' or N2 385 F 5'-ACGACAGATCCAGCAG TAGC-3'; N2 385R 5'-GAGCCTGTTCCATATGTACC$\left.3^{\prime}\right)$. The results of the rtRT-PCR assay designated the influenza virus type A virus to be either subtype H1N1, H3N2 or an unknown virus.

\section{Genomic sequencing and sequence assembly}

The complete nucleotide sequences of 10 viruses were determined using the 454 GS Junior Titanium platform (Roche Applied Science, Indianapolis, IN, USA). In brief, RNA of all viruses was fragmented by $\mathrm{ZnCl}_{2}$ into fragments between $500 \mathrm{bp}$ and $1500 \mathrm{bp}$. Sheared RNA was used for first and second strand cDNA synthesis and fragment end repair. The 454 rapid library multiplex identifier (RL MID) was ligated to the fragments according to the GS Junior Titanium cDNA rapid library preparation method (Roche) by incubating with ligase at $25 \mathrm{C}$ - for 10 min. Quality assessment of the RNA samples and of the DNA library was performed using the FlashGel system. The nucleotide sequence reads obtained were assembled using the Newbler (version 2.5p1) de novo sequence assembly software (Roche). Comparison of the de novo contigs by BLAST with known influenza A virus sequences allowed identification of all genes. Sanger sequencing applying targeted oligo primers was utilized to walk across gaps. BLASTN and BLASTX analyses were performed to compare the established sequences to known influenza A viruses in the NCBI database.

\section{Sequence analysis}

To determine gene relatedness of each gene segment of the Ontario swine H3N2 isolates, we used BLAST from the GenBank database. Geneious Pro 5.5.6. has been used to determine nucleotide and amino acid (aa) sequence identities. The CLUSTAL W alignment method was applied, and an unrooted phylogenetic tree of the HA gene was constructed by using the distance-based neighborjoining method. To predict N-linked glycosylation sites (Asn-X-Ser/Thr, where $\mathrm{X}$ is any aa except Pro) we applied the NetNGlyc 1.0 server.

The University of Guelph Animal Care Committee and the Ethics Committee approved this study.

\section{Additional files}

\section{Additional file 1: Genome constellations identified in Ontario H3N2 viruses isolated from swine in 2011 and 2012. Green $=t r H 3 N 2$. Red = pandemic H1N1 \\ Additional file 2: Phylogenetic trees for the NA gene and the six internal gene segments (A) PB2; (B) PB1; (C) PA; (D) NP; (E) NA; (F) $M ;$ (G) NS of 10 Ontario H3N2 viruses. \\ Additional file 3: Phylogenetic analysis of 32 PB1 sequences. Representatives of the nine PB1 lineages ranging from $\mathrm{A}$ to I have been selected. The pandemic strains of 1918 (H1N1), 1957 (H2N2), 1968 (H3N2) and 2009 (H1N1) are marked with asterisk.}

\section{Competing interests}

All of the authors declared that they have no competing interests with respect to the publication of this manuscript.

\section{Authors' contributions}

$H G$, ZP were involved in project conception, sample collection and data analysis. $H G, M C$ were involved in sequencing. All authors contributed to the writing of this manuscript. All authors read and approved the final manuscript.

\section{Acknowledgments}

The authors of the study are thankful to swine producers and herd veterinarians who participated in the study. The study was financially supported by the Natural Sciences and Engineering Research Council of Canada and Zoetis, through the Collaborative Research and Development Grant funding scheme; and by the OMAFRA-University of Guelph Partnership through the Production Systems research theme.

\section{Author details}

${ }^{1}$ Department of Population Medicine, Ontario Veterinary College, University of Guelph, Guelph, Ontario N1G 2 W1, Canada. ${ }^{2}$ Department of Pathobiology, Ontario Veterinary College, University of Guelph, Guelph, Ontario N1G 2 W1, Canada. ${ }^{3}$ Animal Health Laboratory, University of Guelph, 
Guelph, Ontario N1H 6R8, Canada. ${ }^{4}$ South-West Ontario Veterinary Services, Stratford, Ontario, Canada. ${ }^{5}$ Centre for Public Health and Zoonoses, University of Guelph, Guelph, Canada.

Received: 14 May 2014 Accepted: 29 October 2014

Published online: 22 November 2014

\section{References}

1. Shaw ML, Palese P: Orthomyxoviruses. In Field Virology. 6th edition. Edited by Knipe DM, Howley PM. Philahelphia, PA, USA: Lippincott Williams \& Wilkins; 2014:1151-1185.

2. Tong S, Zhu X, Li Y, Shi M, Zhang J, Bourgeois M, Yang H, Chen X, Recuenco S, Gomez J, Chen LM, Johnson A, Tao Y, Dreyfus C, Yu W, McBride R, Carney PJ, Gilbert AT, Chang J, Guo Z, Davis CT, Paulson JC, Stevens J, Rupprecht CE, Holmes EC, Wilson IA, Donis RO: New world bats harbor diverse influenza A viruses. PLoS Pathog 2013, 9(10):e1003657.

3. Olsen CW, Karasin Al, Carman S, Li Y, Bastien N, Ojkic D, Alves D, Charbonneau G, Henning BM, Low DE, Burton L, Broukhanski G: Triple reassortant H3N2 influenza A viruses, Canada, 2005. Emerg Infect Dis 2006, 12(7):1132-1135.

4. Gagnon CA, Spearman G, Hamel A, Godson DL, Fortin A, Fontaine G, Tremblay D: Characterization of a Canadian mink H3N2 influenza A virus isolate genetically related to triple reassortant swine influenza virus. J Clin Microbiol 2009, 47(3):796-799.

5. Anonymous: Canadian Hog Farms. [http://www.cpc-ccp.com/ canadian hog farms.php] Accessed 2014/09/06

6. Berhane $\mathrm{Y}$, Kehler H, Handel K, Hisanaga T, Xu W, Ojkic D, Pasick J: Molecular and antigenic characterization of reassortant H3N2 viruses from turkeys with a unique constellation of pandemic H1N1 internal genes. PLoS One 2012, 7(3):e32858.

7. Stray SJ, Pittman LB: Subtype- and antigenic site-specific differences in biophysical influences on evolution of influenza virus hemagglutinin. Virol J 2012, 9:91-422X-9-91.

8. Marjuki H, Scholtissek C, Franks J, Negovetich NJ, Aldridge JR, Salomon R, Finkelstein D, Webster RG: Three amino acid changes in PB1-F2 of highly pathogenic $\mathrm{H} 5 \mathrm{~N} 1$ avian influenza virus affect pathogenicity in mallard ducks. Arch Virol 2010, 155(6):925-934.

9. Subbarao EK, London W, Murphy BR: A single amino acid in the PB2 gene of influenza A virus is a determinant of host range. J Virol 1993, 67(4):1761-1764

10. Hatta M, Gao P, Halfmann P, Kawaoka Y: Molecular basis for high virulence of Hong Kong H5N1 influenza A viruses. Science 2001, 293(5536):1840-1842.

11. Shinya K, Hamm S, Hatta M, Ito H, Ito T, Kawaoka Y: PB2 amino acid at position 627 affects replicative efficiency, but not cell tropism, of Hong Kong H5N1 influenza A viruses in mice. Virology 2004, 320(2):258-266.

12. Webby RJ, Swenson SL, Krauss SL, Gerrish PJ, Goyal SM, Webster RG Evolution of swine H3N2 influenza viruses in the United States. J Virol 2000, 74(18):8243-8251.

13. Zhou NN, Senne DA, Landgraf JS, Swenson SL, Erickson G, Rossow K, Liu L, Yoon K, Krauss S, Webster RG: Genetic reassortment of avian, swine, and human influenza A viruses in American pigs. J Virol 1999, 73(10):8851-8856.

14. Tremblay D, Allard V, Doyon JF, Bellehumeur C, Spearman JG, Harel J, Gagnon CA: Emergence of a new swine H3N2 and pandemic (H1N1) 2009 influenza A virus reassortant in two Canadian animal populations, mink and swine. J Clin Microbiol 2011, 49(12):4386-4390.

15. Novel Swine-Origin Influenza A (H1N1) Virus Investigation Team, Dawood FS, Jain S, Finelli L, Shaw MW, Lindstrom S, Garten RJ, Gubareva LV, Xu X, Bridges $C B$, Uyeki TM: Emergence of a novel swine-origin influenza $A$ (H1N1) virus in humans. N Engl J Med 2009, 360(25):2605-2615.

16. Howden KJ, Brockhoff EJ, Caya FD, McLeod L, Lavoie M, Ing JD, Bystrom $J \mathrm{M}$, Alexandersen S, Pasick JM, Berhane Y, Morrison ME, Keenliside JM, Laurendeau S, Rohonczy EB: An investigation into human pandemic influenza virus (H1N1) 2009 on an Alberta swine farm. Can Vet J 2009, 50(11):1153-1161.

17. Berhane Y, Ojkic D, Neufeld J, Leith M, Hisanaga T, Kehler H, Ferencz A, Wojcinski H, Cottam-Birt C, Suderman M, Handel K, Alexandersen S, Pasick J: Molecular characterization of pandemic H1N1 influenza viruses isolated from turkeys and pathogenicity of a human $\mathrm{pH} 1 \mathrm{~N} 1$ isolate in turkeys. Avian Dis 2010, 54(4):1275-1285.

18. Pasma T, Joseph T: Pandemic (H1N1) 2009 infection in swine herds, Manitoba, Canada. Emerg Infect Dis 2010, 16(4):706-708.
19. Nfon CK, Berhane Y, Hisanaga T, Zhang S, Handel K, Kehler H, Labrecque O, Lewis NS, Vincent AL, Copps J, Alexandersen S, Pasick J: Characterization of H1N1 swine influenza viruses circulating in Canadian pigs in 2009. J Virol 2011, 85(17):8667-8679.

20. Ducatez MF, Hause B, Stigger-Rosser E, Darnell D, Corzo C, Juleen K, Simonson R, Brockwell-Staats C, Rubrum A, Wang D, Webb A, Crumpton JC, Lowe J, Gramer M, Webby RJ: Multiple reassortment between pandemic (H1N1) 2009 and endemic influenza viruses in pigs, United States. Emerg Infect Dis 2011, 17(9):1624-1629

21. Kitikoon P, Nelson Ml, Killian ML, Anderson TK, Koster L, Culhane MR, Vincent AL: Genotype patterns of contemporary reassorted H3N2 virus in U.S. swine. J Gen Virol 2013, 94(6):1236-1241.

22. Bowman SA, Sreevatsan S, Killian ML, Page SL, Nelson SW, Nolting JM, Cardona C, Slemons RD: Molecular evidence for interspecies transmission of $\mathrm{H} 3 \mathrm{~N} 2 \mathrm{pM} / \mathrm{H} 3 \mathrm{~N} 2 \mathrm{v}$ influenza A viruses at an Ohio agricultural fair, July 2012. Emerg Microb Infect 2012, 1(e33); doi:10.1038/emi.2012.33

23. Bowman AS, Nelson SW, Page SL, Nolting JM, Killian ML, Sreevatsan $S$, Slemons RD: Swine-to-human transmission of Influenza A(H3N2) virus at agricultural fairs, Ohio, USA, 2012. Emerg Infect Dis 2014, 20(9):1472-1480.

24. Wong KK, Greenbaum A, Moll ME, Lando J, Moore EL, Ganatra R, Biggerstaff M, Lam E, Smith EE, Storms AD, Miller JR, Dato V, Nalluswami K, Nambiar A, Silvestri SA, Lute JR, Ostroff S, Hancock K, Branch A, Trock SC, Klimov A, Shu B, Brammer L, Epperson S, Finelli L, Jhung MA: Outbreak of influenza A (H3N2) variant virus infection among attendees of an agricultural fair, Pennsylvania, USA, 2011. Emerg Infect Dis 2012, 18(12):1937-1944.

25. Pearce MB, Jayaraman A, Pappas C, Belser JA, Zeng H, Gustin KM, Maines TR, Sun X, Raman R, Cox NJ, Sasisekharan R, Katz JM, Tumpey TM: Pathogenesis and transmission of swine origin $\mathrm{A}(\mathrm{H} 3 \mathrm{~N} 2) \mathrm{v}$ influenza viruses in ferrets. Proc Natl Acad Sci U S A 2012, 109(10):3944-3949.

26. Cauchemez S, Epperson S, Biggerstaff M, Swerdlow D, Finelli L, Ferguson NM: Using routine surveillance data to estimate the epidemic potential of emerging zoonoses: application to the emergence of US swine origin influenza A H3N2v virus. PLoS Med 2013, 10(3):e1001399.

27. Wilson IA, Cox NJ: Structural basis of immune recognition of influenza virus hemagglutinin. Annu Rev Immunol 1990, 8:737-771.

28. Wood JM, Oxford JS, Dunleavy U, Newman RW, Major D, Robertson JS: Influenza A ( $\mathrm{H} 1 \mathrm{~N} 1)$ vaccine efficacy in animal models is influenced by two amino acid substitutions in the hemagglutinin molecule. Virology 1989, 171(1):214-221.

29. Kodihalli S, Justewicz DM, Gubareva LV, Webster RG: Selection of a single amino acid substitution in the hemagglutinin molecule by chicken eggs can render influenza A virus $(\mathrm{H} 3)$ candidate vaccine ineffective. J Virol 1995, 69(8):4888-4897

30. Sun S, Wang Q, Zhao F, Chen W, Li Z: Glycosylation site alteration in the evolution of influenza A (H1N1) viruses. PLoS One 2011, 6(7):e22844.

31. Long J, Bushnell RV, Tobin JK, Pan K, Deem MW, Nara PL, Tobin GJ: Evolution of H3N2 influenza virus in a guinea pig model. PLOS One 2011, 6(7):e20130

32. Mishin VP, Novikov D, Hayden FG, Gubareva LV: Effect of hemagglutinin glycosylation on influenza virus susceptibility to neuraminidase inhibitors. J Virol 2005, 79(19):12416-12424.

33. Vigerust DJ, Ulett KB, Boyd KL, Madsen J, Hawgood S, McCullers JA: $\mathrm{N}$-linked glycosylation attenuates H3N2 influenza viruses. J Virol 2007, 81(16):8593-8600.

34. World Health Organization: WHO manual on animal influenza diagnosis and surveillance. 2002.5 Rev.1. http://www.who.int/csr/resources/ publications/influenza/en/whocdscsrncs20025rev.pdf.

35. Spackman E, Senne DA, Myers TJ, Bulaga LL, Garber LP, Perdue ML, Lohman K, Daum LT, Suarez DL: Development of a real-time reverse transcriptase PCR assay for type $\mathrm{A}$ influenza virus and the avian $\mathrm{H} 5$ and $\mathrm{H} 7$ hemagglutinin subtypes. J Clin Microbiol 2002, 40(9):3256-3260.

doi:10.1186/s12985-014-0194-z

Cite this article as: Grgić et al:: Molecular characterization of H3N2 influenza A viruses isolated from Ontario swine in 2011 and 2012. Virology Journal 2014 11:194. 